\title{
Thermodynamic instability of charged dilaton black holes in AdS spaces
}

\author{
A. Sheykhi ${ }^{1,2 *}$, M. H. Dehghani ${ }^{3 \dagger}$ and S. H. Hendi ${ }^{4 \ddagger}$ \\ 1 Department of Physics, Shahid Bahonar University, P.O. Box 76175-132, Kerman, Iran \\ 2 Research Institute for Astronomy and Astrophysics of Maragha (RIAAM), Maragha, Iran \\ 3 Physics Department and Biruni Observatory, Shiraz University, Shiraz 71454, Iran \\ 4 Physics Department, College of Sciences, Yasouj University, Yasouj 75914, Iran
}

\begin{abstract}
We study thermodynamic instability of a class of $(n+1)$-dimensional charged dilatonic spherically symmetric black holes in the background of anti-de Sitter universe. We calculate the quasilocal mass of the AdS dilaton black hole through the use of the subtraction method of Brown and York. We find a Smarr-type formula and perform a stability analysis in the canonical ensemble and disclose the effect of the dilaton field on the thermal stability of the solutions. Our study shows that the solutions are thermally stable for small $\alpha$, while for large $\alpha$ the system has an unstable phase, where $\alpha$ is a coupling constant between the dilaton and matter field.
\end{abstract}

\section{INTRODUCTION}

Strong motivation for studying thermodynamics of black holes in anti-de Sitter (AdS) spaces arises from the correspondence between the gravitating fields in AdS spacetime and conformal field theory (CFT) living on the boundary of the AdS spacetime [1]. It was argued that the thermodynamics of black holes in AdS spaces can be identified with that of a certain dual CFT in the high temperature limit [2]. With the AdS/CFT correspondence, one can gain some insights into thermodynamic properties and phase structures of strong 't Hooft coupling CFTs by studying thermodynamics of AdS black holes. It is well-known that the Schwarzschild black hole in AdS space is thermodynamically stable for large horizon radius, while it becomes unstable for small horizon radius. That is, there is a phase transition, named Hawking-Page phase transition, between the high temperature black hole phase and low temperature thermal AdS space [3]. It has been explained by Witten [2] that the Hawking-Page phase transition of Schwarzschild black holes in AdS spaces can be identified with confinement/deconfinement transition of the Yang-Mills theory in the AdS/CFT correspondence. It is important to note that for the (locally) AdS black holes with zero or negative constant curvature horizon the Hawking-Page phase transition does not appear

\footnotetext{
*sheykhi@mail.uk.ac.ir

$\dagger$ mhd@shirazu.ac.ir

‡ hendi@mail.yu.ac.ir
} 
and these black holes are always locally stable [4 $[\underline{6}]$.

On the other side, there has been a renewed interest in studying scalar coupled solutions of general relativity ever since new black hole solutions have been found in the context of string theory. The low energy limit of the string theory leads to the Einstein gravity, coupled non-minimally to a scalar dilaton field [7]. The dilaton field couples in a nontrivial way to other fields such as gauge fields and results into interesting solutions for the background spacetime [8-10]. These solutions [8 10], however, are all asymptotically flat. It has been shown that with the exception of a pure cosmological constant, no dilaton de-Sitter or anti-de-Sitter black hole solution exists with the presence of only one Liouville-type dilaton potential [11]. In the presence of one or two Liouvilletype dilaton potential, black hole spacetimes which are neither asymptotically flat nor (anti)-de Sitter $[(\mathrm{A}) \mathrm{dS}]$ have been studied in different setups (see e.g [12-17]). With the combination of three Liouville type dilaton potentials, charged dilaton black hole/string solutions in the background of (A)dS spacetime in four [18] and higher dimensional spacetime [19, 20] have been explored. Such potential may arise from the compactification of a higher dimensional supergravity model [21] which originates from the low energy limit of a background string theory.

In this paper, we would like to study thermodynamic instability of asymptotically AdS dilaton black holes in all higher dimensions. In particular, we shall disclose the effect of the dilaton field on the thermal stability of the solutions. The motivation for studying higher dimensional solutions of Einstein gravity originates from string theory which is believed to be the most promising approach to quantum theory of gravity in higher dimensions. In fact, the first successful statistical counting of black hole entropy in string theory was performed for a five-dimensional black hole [22]. This example provides the best laboratory for the microscopic string theory of black holes. Besides, recently it has been realized that there is a way to make the extra dimensions relatively large and still be unobservable. This is if we live on a three dimensional surface (brane) embedded in a higher dimensional spacetime (bulk) [23, 24]. In such a scenario, all gravitational objects such as black holes are higher dimensional. Furthermore, as mathematical objects, black hole spacetimes are among the most important Lorentzian Ricci-flat manifolds in any dimension. One striking feature of the Einstein equations in more than four dimensions is that many uniqueness properties holding in four dimensions are lost. For instance, four-dimensional black holes are known to possess a number of remarkable features, such as uniqueness, spherical topology, dynamical stability, and the laws of black hole mechanics. One would like to know which of these are peculiar to fourdimensions, and which hold more generally. For a recent review on higher dimensional black holes see [25]. In the light of all mentioned above, it becomes obvious that further study on the 
thermodynamics of higher-dimensional dilaton black holes in AdS spaces is of great interest.

This paper is outlined as follows. In Sec. III, we present the $(n+1)$-dimensional black hole solutions of Einstein-Maxwell-dilaton theory in AdS background. In Sec. III, we obtain the conserved and thermodynamic quantities of the solutions and verify the validity of the first law of black hole thermodynamics. We perform a stability analysis in the canonical ensemble and disclose the effect of the dilaton field on the thermal stability of the solutions in Sec. IV. We summarize our results in Sec. V.

\section{DILATON BLACK HOLES IN ADS SPACES}

The action of $(n+1)$-dimensional $(n \geq 3)$ Einstein-Maxwell-dilaton gravity can be written

$$
S=-\frac{1}{16 \pi} \int d^{n+1} x \sqrt{-g}\left(R-\frac{4}{n-1}(\nabla \Phi)^{2}-V(\Phi)-e^{-4 \alpha \Phi /(n-1)} F_{\mu \nu} F^{\mu \nu}\right),
$$

where $R$ is the scalar curvature, $V(\Phi)$ is a potential for the dilaton field $\Phi$. $\alpha$ is an arbitrary constant governing the strength of the coupling between the dilaton and the Maxwell field, $F_{\mu \nu}=$ $\partial_{\mu} A_{\nu}-\partial_{\nu} A_{\mu}$ is the electromagnetic field tensor and $A_{\mu}$ is the electromagnetic potential. While $\alpha=0$ corresponds to the usual Einstein-Maxwell-scalar theory, $\alpha=1$ indicates the dilatonelectromagnetic coupling that appears in the low energy string action in Einstein's frame. Varying action (11) with respect to the gravitational field $g_{\mu \nu}$, the dilaton field $\Phi$ and the gauge field $A_{\mu}$, yields the following field equations

$$
\begin{gathered}
R_{\mu \nu}=\frac{4}{n-1}\left(\partial_{\mu} \Phi \partial_{\nu} \Phi+\frac{1}{4} g_{\mu \nu} V(\Phi)\right)+2 e^{-4 \alpha \Phi /(n-1)}\left(F_{\mu \eta} F_{\nu}^{\eta}-\frac{1}{2(n-1)} g_{\mu \nu} F_{\lambda \eta} F^{\lambda \eta}\right) \\
\nabla^{2} \Phi=\frac{n-1}{8} \frac{\partial V}{\partial \Phi}-\frac{\alpha}{2} e^{-4 \alpha \Phi /(n-1)} F_{\lambda \eta} F^{\lambda \eta} \\
\nabla_{\mu}\left(e^{-4 \alpha \Phi /(n-1)} F^{\mu \nu}\right)=0 .
\end{gathered}
$$

We assume the $(n+1)$-dimensional spherically symmetric metric has the following form

$$
d s^{2}=-N^{2}(\rho) f^{2}(\rho) d t^{2}+\frac{d \rho^{2}}{f^{2}(\rho)}+\rho^{2} R^{2}(\rho) d \Omega_{n-1}^{2},
$$

where $d \Omega_{n-1}^{2}$ denotes the metric of an unit $(n-1)$-sphere and $N(\rho), f(\rho)$ and $R(\rho)$ are functions of $\rho$ which should be determined. First of all, the Maxwell equations (4) can be integrated immediately, where, for the spherically symmetric spacetime (5), all the components of $F_{\mu \nu}$ are zero except $F_{t \rho}$ :

$$
F_{t \rho}=N(\rho) \frac{q e^{4 \alpha \Phi /(n-1)}}{[\rho R(\rho)]^{n-1}},
$$


where $q$, an integration constant, is the charge parameter of the black hole. Our aim here is to construct exact, $(n+1)$-dimensional black hole solutions of Eqs. (2)-(4) for an arbitrary dilaton coupling parameter $\alpha$. The dilaton potential leading to AdS-like solutions of dilaton gravity has been found recently [19]. For an arbitrary value of $\alpha$ in AdS spaces the form of the dilaton potential $V(\Phi)$ in $n+1$ dimensions is chosen as

$$
\begin{aligned}
V(\Phi)= & \frac{2 \Lambda}{n\left(n-2+\alpha^{2}\right)^{2}}\left\{-\alpha^{2}\left[(n+1)^{2}-(n+1) \alpha^{2}-6(n+1)+\alpha^{2}+9\right] e^{-4(n-2) \Phi /[(n-1) \alpha]}\right. \\
& \left.+(n-2)^{2}\left(n-\alpha^{2}\right) e^{4 \alpha \Phi /(n-1)}+4 \alpha^{2}(n-1)(n-2) e^{-2 \Phi\left(n-2-\alpha^{2}\right) /[(n-1) \alpha]}\right\} .
\end{aligned}
$$

Here $\Lambda$ is the cosmological constant. For later convenience we redefine $\Lambda=-n(n-1) / 2 l^{2}$, where $l$ is the AdS radius of spacetime. It is clear the cosmological constant is coupled to the dilaton in a very nontrivial way. This type of dilaton potential can be obtained when a higher-dimensional theory is compactified to four dimensions, including various supergravity models [21]. In the absence of the dilaton field action (1) reduces to the action of Einstein-Maxwell gravity with cosmological constant. Using metric (5) and the Maxwell field (6), one can show that the system of equations (21)-(3) have solutions of the form

$$
\begin{aligned}
N^{2}(\rho)= & {\left[1-\left(\frac{b}{\rho}\right)^{n-2}\right]^{-\gamma(n-3)}, } \\
f^{2}(\rho)= & \left\{\left[1-\left(\frac{c}{\rho}\right)^{n-2}\right]\left[1-\left(\frac{b}{\rho}\right)^{n-2}\right]^{1-\gamma(n-2)}-\frac{2 \Lambda}{n(n-1)} \rho^{2}\left[1-\left(\frac{b}{\rho}\right)^{n-2}\right]^{\gamma}\right\} \\
& \times\left[1-\left(\frac{b}{\rho}\right)^{n-2}\right]^{\gamma(n-3)}, \\
\Phi(\rho)= & \frac{n-1}{4} \sqrt{\gamma(2+2 \gamma-n \gamma)} \ln \left[1-\left(\frac{b}{\rho}\right)^{n-2}\right], \\
R^{2}(\rho)= & {\left[1-\left(\frac{b}{\rho}\right)^{n-2}\right]^{\gamma} . }
\end{aligned}
$$

Here $c$ and $b$ are integration constants and the constant $\gamma$ is

$$
\gamma=\frac{2 \alpha^{2}}{(n-2)\left(n-2+\alpha^{2}\right)} .
$$

The charge parameter $q$ is related to $b$ and $c$ by

$$
q^{2}=\frac{(n-1)(n-2)^{2}}{2\left(n-2+\alpha^{2}\right)} c^{n-2} b^{n-2} .
$$

According to the Gauss theorem, the electric charge of the black hole is

$$
Q=\frac{1}{4 \pi} \int_{\rho \rightarrow \infty} F_{t \rho} \sqrt{-g} d^{n-1} x=\frac{\Omega_{n-1}}{4 \pi} q
$$


where $\Omega_{n-1}$ is the volume of the unit $(n-1)$-sphere. For $\alpha \neq 0$ the solutions become imaginary for $0<\rho<b$ and therefore we should exclude this region from the spacetime. For this purpose we introduce the new radial coordinate $r$ as

$$
r^{2}=\rho^{2}-b^{2} \Rightarrow d \rho^{2}=\frac{r^{2}}{r^{2}+b^{2}} d r^{2}
$$

With this new coordinate, the above metric becomes

$$
d s^{2}=-N^{2}(r) f^{2}(r) d t^{2}+\frac{r^{2} d r^{2}}{\left(r^{2}+b^{2}\right) f^{2}(r)}+\left(r^{2}+b^{2}\right) R^{2}(r) d \Omega_{n-1}^{2},
$$

where the coordinates $r$ assumes the values $0 \leq r<\infty$, and $N(r), f(r), \Phi(r)$ and $R(r)$ are given by Eqs. (8)-(11) with replacement $\rho=\sqrt{r^{2}+b^{2}}$.

The Kretschmann invariant $R_{\mu \nu \lambda \kappa} R^{\mu \nu \lambda \kappa}$ and the Ricci scalar $R$ diverge at $r=0(\rho=b)$. Thus, $r=0$ is a curvature singularity. It is worthwhile to note that the scalar field $\Phi(\rho)$ and the electromagnetic field $F_{t \rho}$ become zero as $\rho \rightarrow \infty$. It is also notable to mention that these solutions are valid for all values of $\alpha$. When $(\alpha=0=\gamma)$, these solutions describe the $(n+1)$-dimensional asymptotically AdS Reissner-Nordstrom black holes. One should note that the singularity for $\alpha \neq 0$ is null, while it is timelike for $\alpha=0$.

The quasilocal mass of the dilaton AdS black hole can be calculated through the use of the subtraction method of Brown and York (BY) [26]. Such a procedure causes the resulting physical quantities to depend on the choice of reference background. In order to use the BY method the metric should have the form

$$
d s^{2}=-W(\mathcal{R}) d t^{2}+\frac{d \mathcal{R}^{2}}{V(\mathcal{R})}+\mathcal{R}^{2} d \Omega^{2} .
$$

Thus, we should write the metric (5) in the above form. To do this, we perform the following transformation:

$$
\mathcal{R}=\rho\left[1-\left(\frac{b}{\rho}\right)^{n-2}\right]^{\gamma / 2} .
$$

It is a matter of calculations to show that the metric (5) may be written as (16) with the following $W$ and $V$ :

$$
\begin{aligned}
& W(\mathcal{R})=N^{2}(\rho(\mathcal{R})) f^{2}(\rho(\mathcal{R})) \\
& V(\mathcal{R})=f^{2}(\rho(\mathcal{R}))\left(\frac{d \mathcal{R}}{d \rho}\right)^{2}=\left[1+\frac{1}{2}(\gamma(n-2)-2)\left(\frac{b}{\rho}\right)^{n-2}\right]^{2}\left[1-\left(\frac{b}{\rho}\right)^{n-2}\right]^{(\gamma-2)} f^{2}(\rho(\mathcal{R})) .
\end{aligned}
$$


The background metric is chosen to be the metric (16) with

$$
W_{0}(\mathcal{R})=V_{0}(\mathcal{R})=f_{0}^{2}(\rho(\mathcal{R}))= \begin{cases}1+\frac{\rho^{2}}{l^{2}}-\frac{2 \alpha^{2} b \rho}{l^{2}\left(1+\alpha^{2}\right)}+\frac{\alpha^{4} b^{2}}{l^{2}\left(1+\alpha^{2}\right)^{2}} & \text { for } n=3 \\ 1+\frac{\rho^{2}}{l^{2}}-\frac{\alpha^{2} b^{2}}{l^{2}\left(2+\alpha^{2}\right)} & \text { for } n=4 \\ 1+\frac{\rho^{2}}{l^{2}} & \text { for } n \geq 5\end{cases}
$$

As you see from above equation, the solutions for $n=3$ and $n=4$ have not "exact" asymptotic AdS behavior. Because of this point, we cannot use the AdS/CFT correspondence to compute the mass. Indeed, for $n \geq 5$ the metric is exactly asymptotically AdS, while for $n=3,4$ it is approximately asymptotically AdS. This is due to the fact that if one compute the Ricci scalar then it is not equal to $-n(n+1) / l^{2}$. It is well-known that the Ricci scalar for AdS spacetime should have this value (see e.g. [27]). Also, the metrics with $f_{0}^{2}(\rho)$ given by Eq. (17) for $n=3$ and $n=4$ do not satisfy the Einstein equation with the cosmological constant, while an AdS spacetime should satisfy Einstein equation with cosmolgical constant, and an asymptotical AdS should satisfy at infinity. On the other side, at large $\rho$, the metric behaves as $\rho^{2}$ and therefore we used the word "approximately" asymptotically AdS.

To compute the conserved mass of the spacetime, we choose a timelike Killing vector field $\xi$ on the boundary surface $\mathcal{B}$ of the spacetime (16). Then the quasilocal conserved mass can be written as

$$
\mathcal{M}=\frac{1}{8 \pi} \int_{\mathcal{B}} d^{2} \varphi \sqrt{\sigma}\left\{\left(K_{a b}-K h_{a b}\right)-\left(K_{a b}^{0}-K^{0} h_{a b}^{0}\right)\right\} n^{a} \xi^{b},
$$

where $\sigma$ is the determinant of the metric of the boundary $\mathcal{B}, K_{a b}^{0}$ is the extrinsic curvature of the background metric and $n^{a}$ is the timelike unit normal vector to the boundary $\mathcal{B}$. In the context of counterterm method, the limit in which the boundary $\mathcal{B}$ becomes infinite $\left(\mathcal{B}_{\infty}\right)$ is taken, and the counterterm prescription ensures that the action and conserved charges are finite. Although the explicit function $f(\rho(\mathcal{R}))$ cannot be obtained, but at large $\mathcal{R}$ this can be done. Thus, one can calculate the mass through the use of the above modified Brown and York formalism as

$$
M=\frac{\Omega_{n-1}}{16 \pi}(n-1)\left[c^{n-2}+\frac{n-2-\alpha^{2}}{n-2+\alpha^{2}} b^{n-2}\right] .
$$

In the absence of a non-trivial dilaton field $(\alpha=0)$, this expression for the mass reduces to the mass of the $(n+1)$-dimensional asymptotically AdS black hole.

\section{THERMODYNAMICS OF ADS DILATON BLACK HOLE}

In this section we intend to study thermodynamics of dilaton black holes in the background of AdS spaces. The entropy of the dilaton black hole typically satisfies the so called area law of the 
entropy which states that the entropy of the black hole is a quarter of the event horizon area [28]. This near universal law applies to almost all kinds of black holes, including dilaton black holes, in Einstein gravity [29]. It is a matter of calculation to show that the entropy of the dilaton black hole is

$$
S=\frac{\Omega_{n-1} b^{n-1} \Gamma_{+}^{\gamma(n-1) / 2}}{4\left(1-\Gamma_{+}\right)^{(n-1) /(n-2)}}
$$

where

$$
\Gamma=1-\left(\frac{b^{2}}{r^{2}+b^{2}}\right)^{(n-2) / 2},
$$

and $\Gamma_{+}=\Gamma\left(r=r_{+}\right)$in which $r_{+}$is the outer horizon and is related to the parameters $b, c, \Lambda$ and $\alpha$. The Hawking temperature of the dilaton black hole on the outer horizon $r_{+}$, can be calculated using the relation

$$
T=\left.\sqrt{r^{2}+b^{2}} \frac{\left(N^{2} f^{2}\right)^{\prime}}{4 \pi N r}\right|_{r=r_{+}},
$$

where a prime stands for the derivative with respect to $r$. One can easily show that

$T=\frac{\Lambda b(n-2) \Gamma_{+}^{1-\gamma(n-1) / 2}}{2 n(n-1) \pi\left(1-\Gamma_{+}\right)^{1 /(n-2)}}\left\{\frac{n(n-1)\left(1-\Gamma_{+}\right)^{2 /(n-2)}}{2 \Lambda b^{2}}-\frac{n \Gamma_{+}^{\gamma(n-1)-1}}{(n-2)}-\frac{[\gamma(n-1)-1]\left(1-\Gamma_{+}\right)}{\Gamma_{+}^{2-\gamma(n-1)}}\right\}$

We have shown the behavior of $T$ versus $\rho_{+}$in various dimensions in Figs. 1 and 2, From these figures we find out that, independent of the spacetime dimensions, for small values of $\alpha$ and $\rho_{+}$, the temperature may be negative $(T<0)$. In this case we encounter a naked singularity. On the other hand, for an extremal black hole the temperature is zero and horizon is degenerate. In this case $r_{\text {ext }}$ is the positive root of the following equation

$$
3 \Gamma_{\text {ext }}^{2-\gamma(n-1)}\left(1-\Gamma_{\text {ext }}\right)^{(4-n) /(n-2)}-\frac{6 \Lambda}{n(n-1)} b^{2}\left[\frac{n \Gamma_{\text {ext }}}{(n-2)\left(1-\Gamma_{\text {ext }}\right)}+\gamma(n-1)-1\right]=0,
$$

where

$$
\Gamma_{\mathrm{ext}}=1-\left(\frac{b}{\sqrt{r_{\mathrm{ext}}^{2}+b^{2}}}\right)^{n-2}
$$

Finally, for large values of $\alpha$ it is always positive provided $\rho_{+}>b$. Substituting solutions (8)-(11) in Eq. (6) , the electromagnetic field can be simplified as

$$
F_{t r}=\frac{q}{\left(r^{2}+b^{2}\right)^{(n-1) / 2}},
$$

while the corresponding gauge potential $A_{t}$ maybe obtained as

$$
A_{t}=-\frac{q}{(n-2) \rho^{n-2}}
$$




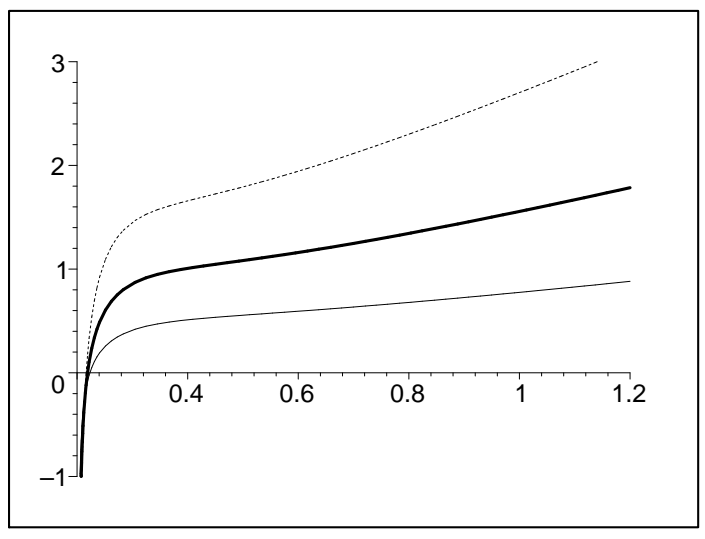

FIG. 1: T versus $\rho_{+}$for $b=0.2, l=1$ and $\alpha=0.1, n=4$ (solid line), $n=5$ (bold line), and $n=6$ (dashed line).

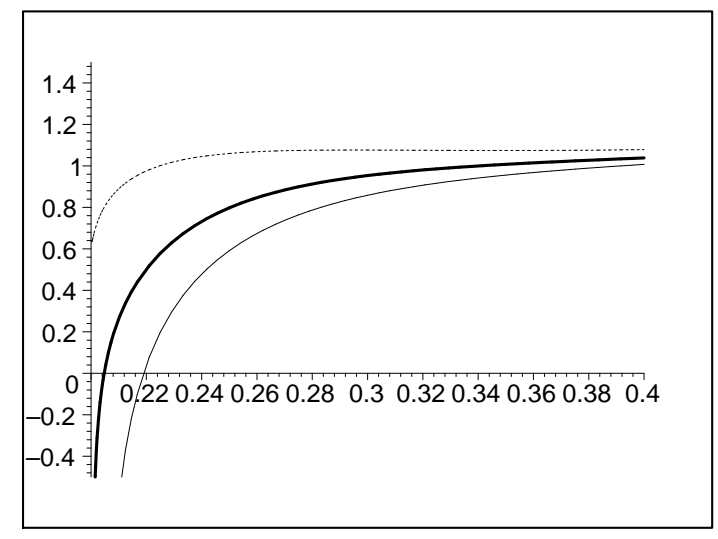

FIG. 2: T versus $\rho_{+}$for $b=0.2, l=1$ and $n=5 . \alpha=0.1$ (solid line), $\alpha=1$ (bold line), and $\alpha=2$ (dashed line).

The electric potential $U$, measured at infinity with respect to the horizon, is defined by [30]

$$
U=\left.A_{\mu} \chi^{\mu}\right|_{r \rightarrow \infty}-\left.A_{\mu} \chi^{\mu}\right|_{r=r_{+}},
$$

where $\chi=\partial_{t}$ is the null generator of the horizon. Therefore, the electric potential may be obtained as

$$
U=\frac{q}{(n-2) \rho_{+}^{n-2}}
$$

where $\rho_{+}^{2}=r_{+}^{2}+b^{2}$. Finally, we check the first law of thermodynamics for the black hole. In order to do this, we obtain the mass $M$ as a function of extensive quantities $S$ and $Q$. Using the expression for the charge, the mass and the entropy given in Eqs. (14), (19) and (20), we can obtain a Smarr-type formula per unit volume as

$$
M(S, Q)=\frac{(n-1)}{16 \pi}\left[\frac{32 \pi^{2}\left(n-2+\alpha^{2}\right) Q^{2} b^{2-n}}{(n-1)(n-2)^{2}}+\frac{n-2-\alpha^{2}}{n-2+\alpha^{2}} b^{n-2}\right],
$$


where $b=b(Q, S)$. One may then regard the parameters $S$ and $Q$ as a complete set of extensive parameters for the mass $M(S, Q)$ and define the intensive parameters conjugate to $S$ and $Q$. These quantities are the temperature and the electric potential

$$
\begin{aligned}
T & =\left(\frac{\partial M}{\partial S}\right)_{Q}=\frac{\left(\frac{\partial M}{\partial b}\right)_{Q}\left(\frac{\partial b}{\partial r_{+}}\right)_{Q}}{\left(\frac{\partial S}{\partial r_{+}}\right)_{Q}+\left(\frac{\partial S}{\partial b}\right)_{Q}\left(\frac{\partial b}{\partial r_{+}}\right)_{Q}} \\
U & =\left(\frac{\partial M}{\partial Q}\right)_{S}+\left(\frac{\partial M}{\partial b}\right)_{S}\left(\frac{\partial b}{\partial Q}\right)_{S},
\end{aligned}
$$

where

$$
\begin{aligned}
& \left(\frac{\partial b}{\partial r_{+}}\right)_{Q}=-\frac{\left(\frac{\partial Z}{\partial r_{+}}\right)_{Q}}{\left(\frac{\partial Z}{\partial b}\right)_{Q}} \\
& Z=\left[1-\frac{32 \pi^{2}\left(n-2+\alpha^{2}\right) Q^{2}}{(n-1)(n-2)^{2} r_{+}^{2 n-4}}\left(\frac{r_{+}}{b}\right)^{n-2}\right]\left[1-\left(\frac{b}{r_{+}}\right)^{n-2}\right]^{1-\gamma(n-2)} \\
& -\frac{2}{n(n-1)} \Lambda r_{+}^{2}\left[1-\left(\frac{b}{r_{+}}\right)^{n-2}\right]^{\gamma} .
\end{aligned}
$$

Straightforward calculations show that the intensive quantities calculated by Eqs. (29) and (30) coincide with Eqs. (22) and (27). Thus, these thermodynamics quantities satisfy the first law of black hole thermodynamics,

$$
d M=T d S+U d Q
$$

\section{STABILITY IN THE CANONICAL ENSEMBLE}

Finally, we study the thermal stability of the solutions in the canonical ensemble. In particular, we will see that the scalar dilaton field makes the solution unstable. The stability of a thermodynamic system with respect to small variations of the thermodynamic coordinates is usually performed by analyzing the behavior of the entropy $S(M, Q)$ around the equilibrium. The local stability in any ensemble requires that $S(M, Q)$ be a concave function of the intensive variables. The stability can also be studied by the behavior of the energy $M(S, Q)$ which should be a convex function of its extensive variable. Thus, the local stability can in principle be carried out by finding the determinant of the Hessian matrix of $M(S, Q)$ with respect to its extensive variables $X_{i}, \mathbf{H}_{X_{i} X_{j}}^{M}=\left[\partial^{2} M / \partial X_{i} \partial X_{j}\right][30,31]$. In our case the mass $M$ is a function of entropy and charge. The number of thermodynamic variables depends on the ensemble that is used. In the canonical ensemble, the charge is a fixed parameter and therefore the positivity of the $\left(\partial^{2} M / \partial S^{2}\right)_{Q}$ is sufficient to ensure local stability. In Fig. 3 we show the behavior of the $\left(\partial^{2} M / \partial S^{2}\right)_{Q}$ as a function of 


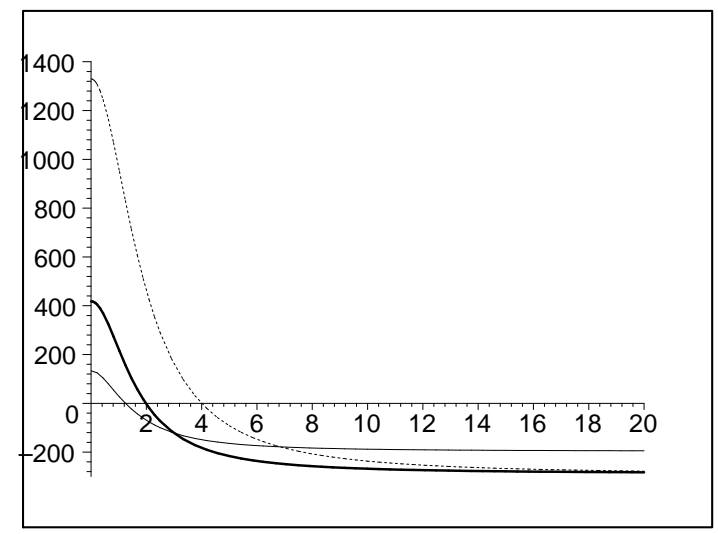

FIG. 3: $\left(\partial^{2} M / \partial S^{2}\right)_{Q}$ versus $\alpha$ for $b=0.2, l=1$ and $\rho_{+}=0.4 ., n=5$ (solid line), $n=6$ (bold line), and $n=7$ (dashed line).

the coupling constant parameter $\alpha$ for different value of $n$. This figure shows that there exists an upper limit for $\alpha$, named $\alpha_{\max }$, for which $\left(\partial^{2} M / \partial S^{2}\right)_{Q}$ is negative provided $\alpha>\alpha_{\max }$ and positive otherwise. That is the black hole solutions are unstable for large values of $\alpha$. It is important to note that $\alpha_{\max }$ depends on the parameters $b, r_{+}$and the dimensionality of spacetime (see Fig. (4). On the other hand, Figs. 5 and 6 show the behavior of the $\left(\partial^{2} M / \partial S^{2}\right)_{Q}$ as a function of the $\rho_{+}$for different value of coupling constant parameter $\alpha$ and different value of $n$. These figures show that for a fixed value of $\alpha$ the solution is thermally stable for $\rho_{+}>b$ in 5,6 and 7 dimension provided $\alpha<\alpha_{\max }$. It is also easy to plot these figures for arbitrary $n$ and generalize the conclusions for higher dimensions.

In comparison with the asymptotically AdS black holes of Einstein gravity, which have a small unstable phase, the stability phase structure of the black holes in Einstein-Maxwell-dilaton gravity shows that the dilaton field crucially changes the stability phase structure.

\section{SUMMARY AND DISCUSSION}

Thermodynamics of black holes in AdS spaces have been the subject of much recent interest. This is primarily due to their relevance for the AdS/CFT correspondence. It was argued that the thermodynamics of black holes in AdS spaces can be identified with that of a certain dual CFT in the high temperature limit. In this paper, we considered asymptotically AdS black holes in $(n+1)$-dimensional Einstein-Maxwell-dilaton gravity. We computed the charge, mass, temperature, entropy and electric potential of the AdS dilaton black holes and found that these quantities satisfy the first law of black hole thermodynamics. We also obtained a Smarr-type formula, $M(S, Q)$, 


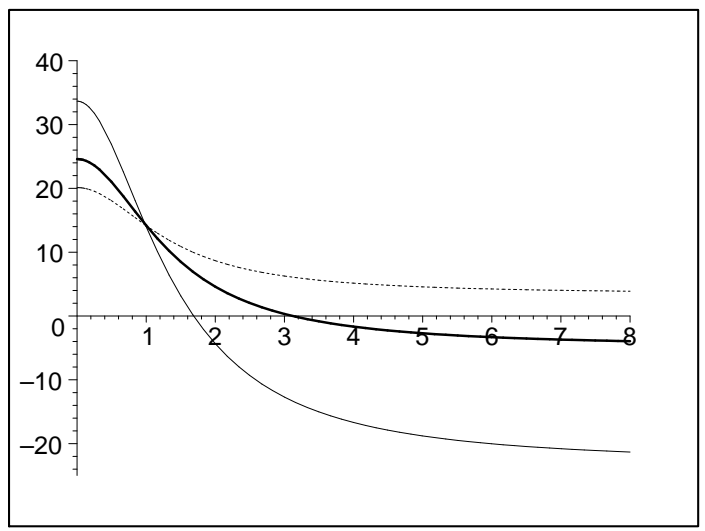

FIG. 4: $\left(\partial^{2} M / \partial S^{2}\right)_{Q}$ versus $\alpha$ for $b=0.2, l=1$ and $n=5 . \rho_{+}=0.5$ (solid line), $\rho_{+}=0.55$ (bold line), and $\rho_{+}=0.6$ (dashed line).

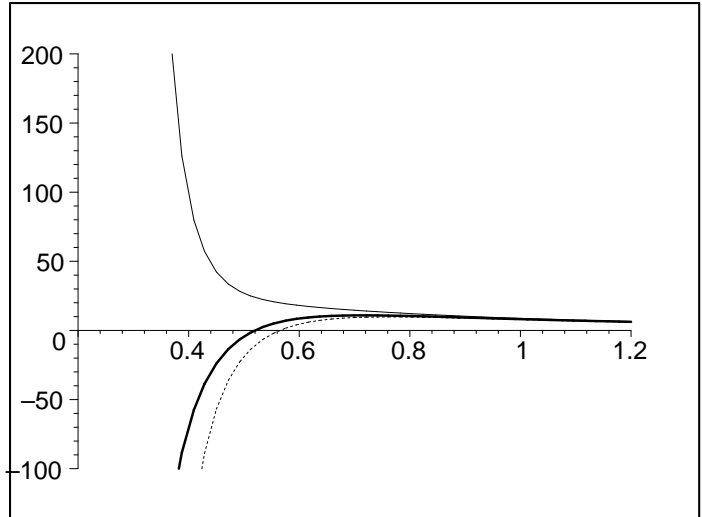

FIG. 5: $\left(\partial^{2} M / \partial S^{2}\right)_{Q}$ versus $\rho_{+}$for $b=0.2, l=1$ and $n=5 . \alpha=0.5$ (solid line), $\alpha=2$ (bold line), and $\alpha=5$ (dashed line).

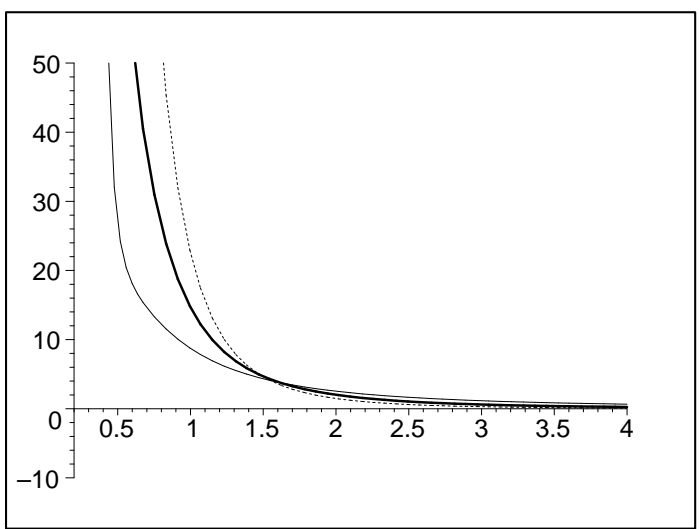

FIG. 6: $\left(\partial^{2} M / \partial S^{2}\right)_{Q}$ versus $\rho_{+}$for $b=0.2, l=1$ and $\alpha=0.5 . n=5$ (solid line), $n=6$ (bold line), and $n=7$ (dashed line). 
and performed a stability analysis in the canonical ensemble by considering $\left(\partial^{2} M / \partial S^{2}\right)_{Q}$ for the charged black hole solutions in $(n+1)$ dimensions and showed that there is no Hawking-Page phase transition in spite of charge of the black holes for small $\alpha$, while the solutions have an unstable phase for large values of $\alpha$. Indeed, for fixed values of the metric parameters, we found that there exists a maximum value of $\alpha$ for which the solutions are thermally unstable if $\alpha>\alpha_{\max }$, where $\alpha_{\max }$ depends on the dimensionality of the spacetime and the metric parameters $b$ and $r_{+}$. This phase behavior shows that although there is no Hawking-Page transition for black object whose horizon is diffeomorphic to $\mathbb{R}^{p}$ for small $\alpha$ and therefore the system is always in the high temperature phase [2], but in the presence of dilaton with $\alpha>\alpha_{\max }$ the black hole solutions have some unstable phases.

Finally, we would like to mention that although charged AdS black holes in dilaton gravity are thermodynamically unstable for large values of dilaton coupling constant $\alpha$, it is worthwhile to examine the dynamical (gravitational) instability of these dilaton black holes. This is due to the fact that there are black holes in Einstein gravity which are thermodynamically unstable, while they are dynamically stable [32]. However, there may be some correlations between the dynamic and thermodynamic instability of black hole solutions in dilaton gravity [31].

\section{Acknowledgments}

We thank the anonymous referees for constructive comments. This work has been supported by Research Institute for Astronomy and Astrophysics of Maragha, Iran.

[1] E. Witten, Adv. Theor. Math. Phys. 2, 253 (1998);

J. M. Maldacena, Adv. Theor. Math. Phys. 2, 231 (1998).

[2] E. Witten, Adv. Theor. Math. Phys. 2, 505 (1998).

[3] S. W. Hawking and D. N. Page, Commun. Math. Phys. 87, 577 (1983).

[4] D. Birmingham, Class. Quant. Grav. 16, 1197 (1999).

[5] D. R. Brill, J. Louko and P. Peldan, Phys. Rev. D 56, 3600 (1997);

A. Chamblin, R. Emparan, C. V. Johnson and R. C. Myers, Phys. Rev. D 60, 064018 (1999).

[6] M. H. Dehghani and S. H. Hendi, Int. J. Mod. Phys. D 16, 1829 (2007);

M. H. Dehghani, N. Alinejadi and S. H. Hendi, Phys. Rev. D 77, 104025 (2008).

[7] M. B. Green, J. H. Schwarz and E. Witten, Superstring Theory, (Cambridge University Press, Cambridge 1987). 
[8] G. W. Gibbons and K. Maeda, Nucl. Phys. B 298, 741 (1988);

T. Koikawa and M. Yoshimura, Phys. Lett. B 189, 29 (1987);

D. Brill and J. Horowitz, Phys. Lett. B 262, 437 (1991).

[9] D. Garfinkle, G. T. Horowitz and A. Strominger, Phys. Rev. D 43, 3140 (1991);

R. Gregory and J. A. Harvey, Phys. Rev. D 47, 2411 (1993).

[10] G. T. Horowitz and A. Strominger, Nucl. Phys. B 360, 197 (1991).

[11] S. J. Poletti, D. L. Wiltshire, Phys. Rev. D 50, 7260 (1994);

S. J. Poletti, J. Twamley and D. L. Wiltshire, Phys. Rev. D 51, 5720 (1995);

S. Mignemi and D. L. Wiltshire, Phys. Rev. D 46, 1475 (1992).

[12] K. C. K. Chan, J. H. Horne and R. B. Mann, Nucl. Phys. B 447, 441 (1995).

[13] R. G. Cai, J. Y. Ji and K. S. Soh, Phys. Rev D 57, 6547 (1998).

[14] G. Clement, D. Gal'tsov and C. Leygnac, Phys. Rev. D 67, 024012 (2003).

[15] A. Sheykhi, M. H. Dehghani, N. Riazi, Phys. Rev. D 75, 044020 (2007);

A. Sheykhi, M. H. Dehghani, N. Riazi and J. Pakravan Phys. Rev. D 74, 084016 (2006);

A. Sheykhi, N. Riazi, Phys. Rev. D 75, 024021 (2007).

[16] A. Sheykhi, Phys. Rev. D 76, 124025 (2007);

A. Sheykhi, Phys. Lett. B 662, 7 (2008).

[17] M. H. Dehghani, J. Pakravan and S. H. Hendi, Phys. Rev. D 74, 104014 (2006);

M. H. Dehghani, et al., J. Cosmol. Astropart. Phys. 02, 020 (2007);

S. H. Hendi, J. Math. Phys. 49, 082501 (2008).

[18] C. J. Gao, S. N. Zhang, Phys. Rev. D 70, 124019 (2004);

A. Sheykhi, Phys. Rev. D 78, 064055 (2008);

A. Sheykhi, Phys. Lett. B 672, 101 (2009).

[19] C. J. Gao, S. N. Zhang, Phys. Lett. B 605, 185 (2005);

C. J. Gao, S. N. Zhang, Phys. Lett. B 612127 (2005).

[20] A. Sheykhi, M. Allahverdizadeh, Phys. Rev. D 78, 064073 (2008);

A. Sheykhi, M. Allahvedizadeh, Gen. Rel. Grav. in press (2009);

A. Sheykhi, M. M. Yazdanpanah, Phys. Lett. B 679, 311 (2009).

[21] S. B. Giddings, Phys. Rev. D 68, 026006 (2003);

E. Radu, D. H. Tchrakian, Class. Quantum Grav. 22, 879 (2005).

[22] A. Strominger and C. Vafa, Phys. Lett. B 379, 99 (1996).

[23] L. Randall, R. Sundrum, Phys. Rev. Lett. 83, 3370 (1999);

L. Randall, R. Sundrum, Phys. Rev. Lett. 83, 4690 (1999).

[24] G. Dvali, G. Gabadadze, M. Porrati, Phys. Lett. B 485, 208 (2000);

G. Dvali, G. Gabadadze, Phys. Rev. D 63, 065007 (2001).

[25] R. Emparan and H. S. Reall, arXive:0801.3471.

[26] J. Brown and J. York, Phys. Rev. D 47, 1407 (1993); 
J.D. Brown, J. Creighton, and R. B. Mann, Phys. Rev. D 50, 6394 (1994).

[27] S. Weinberg, Gravitation and cosmology, (John Wiley 1972, Chapter 15).

[28] J. D. Beckenstein, Phys. Rev. D 7, 2333 (1973);

S. W. Hawking, Nature (London) 248, 30 (1974);

G. W. Gibbons and S. W. Hawking, Phys. Rev. D 15, 2738 (1977).

[29] C. J. Hunter, Phys. Rev. D 59, 024009 (1999);

S. W. Hawking, C. J Hunter and D. N. Page, Phys. Rev. D 59, 044033 (1999);

R. B. Mann Phys. Rev. D 60, 104047 (1999).

[30] M. Cvetic and S. S. Gubser, J. High Energy Phys. 04, 024 (1999);

M. M. Caldarelli, G. Cognola and D. Klemm, Class. Quant. Gravit. 17, 399 (2000).

[31] S. S. Gubser and I. Mitra, J. High Energy Phys. 08, 018 (2001).

[32] R. A. Konoplya and A. Zhidenko, Phys. Rev. D 78, 104017 (2008);

D. Birmingham and S. Mokhtari, Phys. Rev. D 76, 124039 (2007). 